\title{
Interferon Gamma Release Assays in active Tuberculosis: new medical insights
}

\author{
Sandro Pierdomenico', Sergio Gallazzi', Daniela De Francesco', Carla Cisari',Amedea Giani' \\ I Laboratorio Analisi, A.O. Ospedale di Circolo di Busto Arsizio \\ 2 Laboratorio di Microbiologia, Ospedale di Circolo e Fondazione Macchi, Varese
}

Key words: IGRA (Interferon-Gamma Release Assay), ESAT-6 (Early Secretory Antigen 6), CFP-I0 (Culture Filtrate Protein 10), TST (Tubercolin Skin Test), LTBI (Latent Tuberculosis Infection).

IGRAs (Interferon-Gamma Release Assay) nella Tubercolosi attiva: nuove intuizioni mediche

\section{SUMMARY}

Since first presentation, Interferon $\gamma$ Release Assays (IGRAs) have had basic and wide application to LTBI, in accordance with international consensus and CDC recommendations, leaving their use in active TB to the field of study and research. We reviewed the results of 633 patients investigated from 2004 to 2008 targeting active $\mathrm{TB}$, with the objective to highlight immunological data supporting test performances. We evaluated Quantiferon TB Gold ( $\left.\right|^{\text {st }}$ generation IGRA kit) in association to Culture (MGIT 960 and Lowenstein Jensen) and PCR (Probetec-ET) having the positivity of culture plus clinical diagnosis as the standard true value to compare.

QTB Gold was studied in 69 TB positive patients (42 pulmonary and 27 extra-pulmonary), with Sensitivity, Specificity, PPV and NPV average to $61.8 \%, 94.5 \%, 54.3 \%$ and $95.9 \%$ respectively, after indeterminate results discharging. Significant statistical differences didn't emerge between pulmonary and extra-pulmonary infections (Cl 95\%). The overall indeterminate ratio arose up to $20.3 \%$ in patients with active TB vs $2.7 \%$ of global population $(\mathrm{p}<0.00 \mathrm{I})$. In $22 \%$ of patients with active pulmonary disease, IGRA conversed to positivity after I5 days in replicated tests, in spite of current treatment. 4 patients, with pulmonary TB and Quantiferon persistent negativities, underwent 18 months follow-up as not respondent although SIRE phenotypic susceptibilities and enough DOT compliance. Molecular DST documented hetero resistance for rpoB (MUT I, MUT 3 plus wild lines) and katG (MUT I plus wild) in association to lack of inhA wild lines (Genotype MTBDR plus, Hain Lifescience). These reports suggest a mutational relationship between Rv3874 - 3875 cassette, encoding ESAT-6 / CFP-I0, and rpoB, katG, inhA genes plausibly implying weak or absent selective clonal Th I activation to IGRA antigens.

Our data seem to point out: I) positive results are able to match true active TB in less than $50 \%$ of patients; 2 ) negative results could leave undiagnosed or misdiagnosed more than $30 \%$ of active TB; 3 ) the higher level of indeterminate results and the conversing positivity in replicated IGRA tests might find their root either in Mycobacteria genome and microbiome variability or in the lymphocyte kinetic and APCs/DCs inhibition due to inflammatory engage, present in human active TB and already focused in animal model, with IL- I0 key role like in T-Cell exhaustion phenotype.

\section{INTRODUZIONE}

Come premessa ad un articolo sulla Tubercolosi è usuale definirne l'impatto epidemiologico: $1 / 3$ dell'umanità coinvolta dall'endemia; $10200000 /$ anno i nuovi casi in forma attiva, con 2300000 decessi/anno dal 2005 al 2007 (1, 9, 27). La presentazione di un test su sangue, basato sulla misura di Interferon $\gamma$ rilasciato dopo stimolazione di linfociti Th1 effettori, con antigeni puri e specifici per M. tuberculosis (ESAT-6 e CFP-10 e poi TB 7-7), ha colto l'entusiasmo di molti $(20,26,29)$.

Le formulazioni commerciali disponibili sono due: il Quantiferon TB Gold (Cellestis Ltd, Carnegie, Victoria, Australia) ed il T-SPOT.TB (Oxford Immunotec, Oxford, UK). Nel Dicembre 2004 il primo dei test è stato approvato dal FDA e nel Dicembre 2005 il CDC ha formulato 2 linee guida indirizzate all'uso razionale dei metodi IGRA per la diagnosi di LTBI, in sequenza o alternativa al TST nella popolazione generale, in specifiche classi di pazienti e negli operatori sanitari soggetti a rischio occupazionale (17-19).

Già da una Review del 2004 risultavano 42 pubblicazioni sull'argomento. Sono stati completati sia studi di praticabilità clinica e di costo/beneficio dei test IGRA vs il TST nella LTBI e nell'infezione tubercolare attiva, sia studi comparativi dei 2 metodi commerciali, in gruppi campionari selezionati ed in routine $(2,5,8,14,24)$. Tra il 2004 ed il Marzo 2011 sono state emanate 33 linee guida o raccomandazioni, da organismi di 25 nazioni e da 2 organizzazioni sovranazionali (6). 


\section{MATERIALI E METODI}

Il nostro laboratorio opera, per la diagnosi tubercolare, su un bacino d'utenza di 450.000 abitanti nell'hinterland nord ovest di Milano. La densità abitativa è di tipo urbano, il tessuto economico industriale ed un income pro capite superiore ai 30000 Euro/anno. La realtà favorisce un alto indice di immigrazione con prevalente provenienza dall'Africa e dall'Est Europa. L'ambiente e l'immigrazione rendono conto di una incidenza dal 2003 al 2006 da 17.6 a 16.0 nuovi casi di TBC attiva/100000 abitanti vs il 10.4 della Regione Lombardia ed il 9/100000 nazionale $(3,10)$.

Il peso relativo degli immigrati, con residenza $<5$ anni, è del $42 \%$. La diffusione dei ceppi MDR (INH $\mathrm{R}$ e RMP R) è pari al 1.5\% degli isolati/paziente. Gli stipiti XDR sono $<0.5 \%(23,28)$.

Lo studio si estende dal Settembre 2004 ad Aprile 2008; comprende 633 pazienti indagati con microscopia, coltura, PCR e Quantiferon per sospetta TB attiva polmonare o extrapolmonare.

L'obiettivo è determinare il contributo del Quantiferon nella diagnosi dell'infezione tubercolare attiva.

È stato definito come unitario episodio infettivo il soggetto indagato per 8 mesi con più materiali ed in più momenti assistenziali, per escludere duplicazione di dati.

La microscopia è stata condotta con Auramina/Rhodamina e con Ziehl-Neelsen per sovracolorazione in casi dubbi o positivi.

La PCR utilizzata (Probetec ET, Becton Dickinson) ha target IS6110 con rilevazione/amplificazione isoterma a $52.5^{\circ} \mathrm{C}(25)$.

La coltura ha rispettato protocolli del ASM e del AMCLI per le fasi pre-analitica e analitica: decontaminazione con $\mathrm{NaOH}$ al $1.5-2 \%$ finale (Myco-Prep, BD) e inoculo su terreni Lowenstein Jensen, Middle Brook 7H9 e flacone MTB del sistema MGIT 960 (Becton Dickinson, Cockeysville, USA). È stato adottato un sistema di identificazione molecolare, con reattivi Genotype CM e AS (Hain Lifescience, Neheren, Germany) (30).

Il test fenotipico di sensibilità a Rifampicina, Isoniazide, Streptomicina, Etambutolo e Pyrazinamide è stato eseguito con sistema MGIT 960; quello molecolare con metodologia Line Probe Assay Genotype MTBDRplus (Hain Lifescience). I farmaci di II linea sono stati testati, per gradiente di diffusione, con E-test $(\mathrm{AB}$ Biodisk, Solna, Sweden) (12, 15, 22).

Il Controllo di Qualità Interno è stato effettuato con frazione di tampone fosfato della fase di neutralizzazione (QCI-) e con stipite ATCC 27156 (QCI+). La valutazione esterna di qualità ha seguito il programma UKNEQAS "Quality
Assessment Scheme for Mycobacteriology".

Il Quantiferon-TB Gold è stato utilizzato secondo procedura "FDA approved"(9). Il criterio di validazione è basato sulla pubblicazione della Cellestis del Settembre 2004 ed approvato dal FDA il 06 Dicembre 2004.

Non ci siamo orientati, durante lo studio, verso la versione IT (In-Tube) per controllare in laboratorio il rapporto quantitativo sangue - miscela antigenica, per differenziare le risposte ai singoli antigeni (ESAT-6 e CFP-10) e per rispettare gli stessi criteri di validazione durante i 4 anni di osservazione.

\section{RISULTATI}

Dal gruppo di 633 pazienti, la positività per TB attiva è stata documentata in 69 casi $(10.9 \%)$ : 42 polmonari e 27 extra-polmonari. In 68 è stato rispettato il gold standard microbiologico (coltura positiva e correlazione clinica), in 1 caso il gold standard pseudo-clinico (PCR positiva, istologia positiva, coltura negativa e diagnosi di tabe meseraica).

Il Quantiferon-TB Gold è risultato positivo in 62 pazienti, indeterminato in 29 (14 nei soggetti con TB attiva) e negativo in 542 .

Le Tabelle 1 e 2 riassumono la distribuzione dei risultati del test ELISA dopo esclusione degli indeterminati.

Nelle forme polmonari il Quantiferon-TB Gold presenta: Sensibilità $=61.7 \%$, Specificità $=$ 95.5\%, PPV $=56.7 \%$, NPV $=96.3 \%$. Nelle forme extrapolmonari: Sensibilità $=61.9 \%$, Specificità $=$ $93.6 \%, \mathrm{PPV}=52.0 \%$, NPV $=95.6 \%$.

L'analisi non rivela differenze statisticamente significative di prestazioni analitiche e di probabilità di risultato indeterminato tra forme extrapolmonari e polmonari (IC 95\%).

È invece significativa la differenza della percentuale degli indeterminati nei pazienti senza e con TB attiva: $2.7 \%$ vs $20.3 \%$ ( $<<0.001)$.

I 14 casi con risultato indeterminato ( 8 polmonari e 6 extrapolmonari) risultano associati a positività microscopica da $1+$ a $3+$, PCR + e conferma colturale in 12-19 giorni, in assenza di patologie concomitanti e con conta/ tipizzazione linfocitaria normali, su prelievi eseguiti prima della terapia.

In letteratura è prospettato che fino al $25 \%$ dei soggetti, all'esordio della malattia attiva, possa andare incontro ad una attenuazione della risposta immunitaria cellulo-mediata (CMI) prima del trattamento $(13,21,27)$.

Questa interpretazione ci ha indotto a ripetere il prelievo in 9 casi in cui una prima risposta era risultata negativa. In 2 pazienti (di 65 e 74 anni, con positività microscopica da $1+$ a $2+, \mathrm{PCR}+\mathrm{e}$ conferma colturale di TBC polmonare) il secondo 
prelievo eseguito a 7 e 15 giorni dal primo, rispettivamente, ha visto convertire il risultato da negativo a positivo sia per ESAT-6 (da 0.14 e 0.02 a 1.11 e $0.35 \mathrm{IU} / \mathrm{mL}$ ) sia per CFP-10 (da 0.21 a 4.68 $\mathrm{IU} / \mathrm{mL}$ ) nonostante la terapia fosse iniziata da più di 7 giorni.

In 4 dei 13 soggetti con TBC polmonare e Quantiferon negativo è stato effettuato un followup prolungato, con controlli a 4, 6 e 18 mesi, per refrattarietà alla terapia, pur con sensibilità fenotipiche documentate ed adeguata compliance dei pazienti. L'antibiogramma molecolare ha rilevato un pattern di etero resistenza per rpoB (MUT 1 e MUT 3) e katG (MUT 1) con linee Wild assenti per inhA. La costante negatività del QuantiferonTB Gold, in presenza di una potente risposta al PHA, sembra suggerire una relazione mutazionale tra la cassetta genica $\mathrm{Rv} 3874$ e 3875 codificante per ESAT-6 e CFP-10 ed i geni di resistenza rpoB, katG e inhA, con risultante deficit di attivazione clonale Th1 - specifica per gli antigeni IGRA.

\section{DISCUSSIONE}

Il Quantiferon-TB Gold, nella esperienza condotta su 69 pazienti con Tubercolosi microbiologicamente documentata, presenta una sensibilità media del $61.8 \%$ ed un PPV medio del $54 \%$. Più bassi di quanto evidenziato in letteratura (85$89 \%)(20,26)$ e fortemente compromessi dall'alto numero di indeterminati (20.3\%).

Il test sembra rivestire un ruolo solo complementare nella diagnosi della tubercolosi attiva.

La sua praticabilità analitica rimane condizionata da una serie di fattori:

1 - scarsa chiarezza nei criteri di validazione;

2 - alta imprecisione, particolarmente tra le serie;

3 - instabilità dello standard ed alto background analitico;

4 - particolare algoritmo di calcolo delle concentrazioni;

5 - alto numero di indeterminati;

6 - debole reattività antigenica nei pazienti con TB attiva.

L'inibizione della CMI è più problematica della stessa terapia, per la misura in vitro dell'interferon $\gamma$ come risposta secretiva ad antigeni tubercolari. Recentemente il fenomeno è stato descritto come fenotipo comune nelle infezioni da patogeni persistenti (come il Bacillo di Koch) e nel cancro e ricondotto ad un evento denominato " $\mathrm{T}$ cell exhaustion", con ruolo chiave di IL-10 (11).

Tabella I. Forme polmonari

\begin{tabular}{ccc}
\hline $\mathbf{N}^{\circ}$ & TBC + & TBC - \\
\hline Quantiferon + & 21 & 16 \\
\hline Quantiferon - & 13 & 344 \\
\hline
\end{tabular}

Altre significative variabili condizionano l'efficacia diagnostica del Quantiferon-TB Gold: il numero totale di linfociti, di cellule Th1 effettrici ed il livello basale di IFN- $\gamma$ (Nihil), rappresentando questo il "Bianco" della rilevazione Immunoenzimatica. Non a caso nella versione in tube la soglia di validazione del Nihil è stata portata da $0.7 \mathrm{IU} / \mathrm{mL}$ a $8 \mathrm{IU} / \mathrm{mL}(6,24)$.

\section{CONCLUSIONI}

La tubercolosi è una malattia antica che riveste, all'esordio del XXI secolo, un enorme peso epidemiologico e così si prevede rimanga fino al 2020 ed oltre (27).

Le sue caratteristiche: alta contagiosità, bassa dose infettante, diffusa endemia, presenza di stipiti MDR iniziali, aumentata incidenza della resistenza acquisita XDR pongono ancora oggi anche nei paesi del Nord del mondo, il grosso problema della prevenzione e del controllo.

I test IGRA possono rappresentare, in potenza, una risposta al divenire diagnostico e terapeutico così come altri marker di infezione, come la adenosina-deaminasi (16).

In atto, i nostri dati suggeriscono che il Quantiferon-TB Gold quando positivo, con una sensibilità media del $61.8 \%$ ed un PPV del 52$56 \%$, può documentare la presenza tubercolare attiva in meno del $50 \%$ dei casi ed il risultato negativo, associato agli indeterminati, pur con specificità del $93-95 \%$ ed un NPV medio del $95.9 \%$, comporta una probabilità post-test $>30 \%$ di malattia non diagnosticata (assumendo una probabilità pre-test del 10\%).

Nei pazienti con sospetta TB attiva, il rapporto costi/benefici depone quindi per un uso dei test IGRA solo complementare, limitato ai casi con microscopia e PCR negativi e riservando sempre alla coltura il valore di gold standard diagnostico.

\section{BIBLIOGRAFIA}

1. Asgharzadeh M, Kafil HS. Current trends in molecular epidemiology studies of Mycobacterium tuberculosis. Biot Mol Biol Rev 2007; 2 (5): 108-15.

2. Bock I, Weldingh K, Lilleback T, Follmann F, Andersen P. Comparison of tuberculin skin test and new specific blood test in tuberculosis contacts. Am J Resp Crit Care Med 2004; 170: 65-9.

3. Boll Uff Reg Lombardia. $1^{\circ}$ Supplemento straordinario. Piano Socio Sanitario Regionale 2002-2004: prevenzione, sorveglianza e controllo della malattia tubercolare. 4 Gennaio 2005, 3-47.

4. Brosch R, Gordon SV, Marmiesse M, et al. A new evolutionary scenario for the Mycobacterium tuberculosis

Tabella 2. Forme extrapolmonari

\begin{tabular}{ccc}
\hline $\mathbf{N}^{\circ}$ & TBC + & TBC - \\
\hline Quantiferon + & 13 & 12 \\
\hline Quantiferon - & 8 & \\
\hline
\end{tabular}


complex. Proc Natl Acad Sci USA 2002; 99: 3684-9.

5. Colford JM, Pai M, et al. Mycobacterium tuberculosis Infection in health Care Workers in rural India. Comparison of a whole-blood Interferon $\gamma$ Assay with Tuberculin Skin Testing. JAMA, 2005; 293 (22): 2746-55.

6. Denkinger CM, Dheda K, Pai M. Guidelines on interferon- $\gamma$ release assays for tuberculosis infection: concordance, discordance or confusion? Clin Microbiol Infect 2011; 17: 806-14.

7. Ewer K, Deek J, Alvarez L, et al. Comparison of Tcell-based assay with tuberculin skin test for diagnosis of Mycobacterium tuberculosis infection in a school tuberculosis outbreak. The Lancet 2003; 361: 116873.

8. Fietta A, Meloni F, Cascina A, et al. Comparison of a whole-blood interferon- $\gamma$ assay and tuberculin skin testing in patients with active tuberculosis and individuals at high or low risk of Mycobacterium tuberculosis infection. AJIC 2003; 31(6): 347-53.

9. Fitzgerald DW, Sterling TR, Haas DW. Mycobacterium tuberculosis. In: Principles and Practice of Infectious Diseases, Mandell GL, Bennett JE, Dolin R, Seventh Edition. Churchill Livingstone, Philadelphia PA 2010; 3129-63.

10. Garzelli C, Lari N, Cuccu B, Tortoli E, Rindi L. Impact of immigration on tuberculosis in a low-incidence area of Italy: a molecular epidemiological approach. Clin Microbiol Infect 2010; 16(11): 1691-7.

11. Hyun-Tak Jin, Yun Hee Jeong, Hyo Jin Park, Sang-Jun Ha. Mechanism of T cell exhaustion in a chronic environment. BMB reports 2011; 44(4): 217-31.

12. Inderlied CB, Pfyffer GE. Susceptibility test methods: Mycobacteria. In: Murray PR, Baron JO, Jorgensen $\mathrm{JH}$, Pfaller MA, Yolken RH, Eight Edition. American Society for Microbiology, Washington DC 2003; 1149-77.

13. Janeway CA, Travers P, Walport M, Shlomchik M. Immunobiologia Quinta Edizione, Piccin, Padova 2003; 305-52, 395-439.

14. Kang YA, Hye WL, et al. Discrepancy between the tuberculin skin test and the whole-blood Interferon $\gamma$ Assay for the Diagnosis of Latent Tuberculosis Infection in an Intermediate Tuberculosis-Burden Country. JAMA June 8, 2005; 293 (22): 2756-61.

15. Kim SJ. Drug-susceptibility testing in tuberculosis: methods and reliability of results. Eur Resp Jour 2005; 25: 564-9.

16. Liang QL, Shi HZ, Wang K, Qin SM, Qin XJ. Diagnostic accuracy of adenosine deaminase in tuberculous pleurisy: a meta-analysis. Respir Med 2008; 102 (5): 744-54.

17. Mazurek GH, Jereb J, Lobue P, Iademarco MF, Metchock B, Vernon A. Guidelines for using the QuantiFERON-TB Gold test for detecting Mycobacterium tuberculosis infection, United States. MMWR Recomm Rep 2005; 54 (RR-15): 49-55.
18. Mazurek GH, Jereb J, Vernon A, LoBue P, Goldberg $\mathrm{S}$, Castro K. Updated guidelines for using interferon gamma release assays to detect Mycobacterium tuberculosis infection- United States, 2010. MMWR Recomm Rep 2010; 59 (RR-5): 1-25.

19. Mazurek GH, Villarino ME. Guidelines for using Quantiferon-TB test for diagnosing latent Mycobacterium tuberculosis Infection. Morb and Mort WKLY Report 2002; 51:1-7.

20. Mori T, Sakatani M, Yamagishi F, et al. Specific Detection of Tuberculosis Infection with an Interferon-gamma Based Assay Using New Antigens. Am J Resp Crit Care Med 2004; 170 (1): 59-64.

21. Murphy K. Travers P, Walport M. Janeway's Immunobiologia. Settima Edizione. Piccin Nuova Libraria, Padova 2010; 323-42, 421-54.

22. National Committee for Clinical Laboratory Standards. M24-T2 Susceptibility testing of Mycobacteria, Nocardia and other aerobic actynomycetes; tentative standard. Second edition. National Committee for Clinical Laboratory Standards, Villanova PA 2001.

23. Odone A, Ricco M, Morandi M, Borrini BM, Pasquarella C, Signorelli C. Epidemiology of Tuberculosis in a low-incidence italian region with high immigration rates: differences between not Italyborn and Italy-born TB cases. BMC 2011; 11(1): 376 (Epub ahead of print)

24. Pai M, Riley LW, Colford JM jr. Interferon-gamma assays in the immunodiagnosis of tuberculosis: a systematic review. Lancet Infect Dis 2004; 4: 761-76.

25. Pfyffer GE, Brown-Elliott BA, Wallace RJ. Mycobacterium: general characteristics, isolation and staining procedures. In: Murray $\mathrm{PR}$, Baron JO, Jorgensen JH, Pfaller MA, Yolken RH, Eight Edition. American Society for Microbiology, Washington DC 2003; 532-59.

26. Rayn P, Munk ME, et al. Prospective Evaluation of a whole-blood test Using Mycbacterium tuberculosisspecific Antigen ESAT-6 and CFP-10 for diagnosis of Active Tuberculosis. Clin Diagn Lab Immun, Apr 2005; 12: 491-6.

27. Rom WN, Garay SM. Tuberculosis. Second Edition, Lippincot Williams \& Wilkins, Philadelphia PA 2004; 13-29, 47-114, 135-81, 185-320, 427-44.

28. Sougakoff W. Molecular epidemiology of multidrugresistant strains of Mycobacterium tuberculosis. Clin Microbiol Infect 2011; 17: 800-5.

29. Toru M, Mitsunori S, Fumio Y, et al. Specific Detection of Tuberculosis Infection: an Interferon- $\gamma$ based Assay Using new Antigens. Am Jour Resp Crit Care Med 2004; 170: 59-64.

30. Vincent V, Brown-Elliott BA, Jost KC, Wallace RJ jr. Mycobacterium: phenotypic and genotypic identification. In: Murray PR, Baron JO, Jorgensen JH, Pfaller MA, Yolken RH, Eight Edition. American Society for Microbiology, Washington DC 2003; 560-84. 